Physics

Physics Research Publications

\title{
Nonlinear magnetization behavior near the spin-glass transition in the layered III-VI diluted magnetic semiconductor Ga1-xMnxS
}
T. M. Pekarek
E. M. Watson
J. Garner
P. M. Shand
I. Miotkowski
A. K. Ramdas

This paper is posted at Purdue e-Pubs.

http://docs.lib.purdue.edu/physics_articles/688 


\title{
Nonlinear magnetization behavior near the spin-glass transition in the layered III-VI diluted magnetic semiconductor $\mathrm{Ga}_{1-x} \mathrm{Mn}_{x} \mathrm{~S}$
}

\author{
T. M. Pekarek, ${ }^{\text {a) }}$ E. M. Watson, and J. Garner \\ Department of Chemistry and Physics, University of North Florida, Jacksonville, Florida 32224 \\ P. M. Shand \\ Department of Physics, University of Northern Iowa, Cedar Falls, Iowa 50614
}

I. Miotkowski and A. K. Ramdas

Department of Physics, Purdue University, West Lafayette, Indiana 47907

(Presented on 11 January 2007; received 31 October 2006; accepted 2 December 2006; published online 30 April 2007)

\begin{abstract}
Magnetic properties of single crystalline $\mathrm{Ga}_{1-x} \mathrm{Mn}_{x} \mathrm{~S} \quad(x=0.09)$ have been measured near the spin-glass transition. No other III-VI DMS is currently known to exhibit a spin-glass transition for comparison with $\mathrm{Ga}_{1-x} \mathrm{Mn}_{x} \mathrm{~S}$. $\mathrm{Ga}_{1-x} \mathrm{Mn}_{x} \mathrm{~S}\left(T_{c}=11.2 \mathrm{~K}\right)$ shows unexpected similarity to II-VI DMS $\mathrm{Zn}_{1-x} \mathrm{Mn}_{x} \mathrm{Te}\left(T_{c}=20.8 \mathrm{~K}\right)$. For both systems, the quantity $\chi_{\mathrm{nl}}=-M_{\mathrm{nl}} / H$ (where $\left.M_{\mathrm{nl}}=M_{\mathrm{total}}-M_{\text {linear }}\right)$ diverges as the temperature approaches the spin-glass transition temperature $T_{c}$ from above. It also increases with applied field at temperatures just above $T_{c}$ for both systems. The spin-glass transitions in both $\mathrm{Ga}_{1-x} \mathrm{Mn}_{x} \mathrm{~S}$ and $\mathrm{Zn}_{1-x} \mathrm{Mn}_{x}$ Te follow the same universal scaling function with the same critical exponent values $(\gamma=4.0 \pm 1.0$ and $\beta=0.8 \pm 0.2)$. The temperature of $\mathrm{Ga}_{1-x} \mathrm{Mn}_{x} \mathrm{~S}$ 's low-field spin-glass transition cusp was found to occur at $10.9 \mathrm{~K}$. As expected, this is slightly lower than the true spin-glass transition temperature $T_{c}=11.2 \pm 0.2 \mathrm{~K}$ obtained from the scaling analysis. The asymptotic, zero-field limit of the spin-glass transition cusp was found to persist at $10.9 \mathrm{~K}$ up to $\sim 1 \mathrm{~T}$ before being suppressed to lower temperatures with increasing field. Questions remain about if $\mathrm{Ga}_{1-x} \mathrm{Mn}_{x} \mathrm{~S}$ should belong in the same universality class as $\mathrm{Zn}_{1-x} \mathrm{Mn}_{x}$ Te since $\mathrm{Ga}_{1-x} \mathrm{Mn}_{x} \mathrm{~S}$ is quasi-two-dimensional and $\mathrm{Zn}_{1-x} \mathrm{Mn}_{x} \mathrm{Te}$ is three-dimensional. (C) 2007 American Institute of Physics. [DOI: 10.1063/1.2710340]
\end{abstract}

\section{INTRODUCTION}

$\mathrm{Ga}_{1-x} \mathrm{Mn}_{x} \mathrm{~S}$ is in the class of layered III-VI diluted magnetic semiconductors (DMSs). The two-dimensional crystal structure of $\mathrm{Ga}_{1-x} \mathrm{Mn}_{x} \mathrm{~S}$ (inset of Fig. 1) is confined by van der Waals bonds above and below the four atom thick layer Superexchange between two Mn ions is mediated by a S ion in the top and bottom capping layers (Mn-S-Mn) similar to the II-VI DMS. However, more complex exchange channels such as $\mathrm{Mn}-\mathrm{S}-\mathrm{Ga}-\mathrm{Mn}$ or direct $\mathrm{Mn}-\mathrm{Mn}$ bonds are available in the III-VI DMS systems.

Only a few III-VI DMS systems have been investigated to date: $\mathrm{Ga}_{1-x} \mathrm{Mn}_{x} \mathrm{~S},{ }^{1-3} \mathrm{In}_{1-x} \mathrm{Mn}_{x} \mathrm{~S},{ }^{4,5} \mathrm{In}_{1-x} \mathrm{Mn}_{x} \mathrm{Se},{ }^{6,7}$ $\mathrm{Ga}_{1-x} \mathrm{Mn}_{x} \mathrm{Se},{ }^{8}$ and $\mathrm{Ga}_{1-x} \mathrm{Fe}_{x} \mathrm{Se}^{9} \mathrm{Ga}_{1-x} \mathrm{Mn}_{x} \mathrm{~S}$ and $\mathrm{In}_{1-x} \mathrm{Mn}_{x} \mathrm{~S}$ are reasonably well understood at this time. In $\mathrm{Ga}_{1-x} \mathrm{Mn}_{x} \mathrm{~S}$ and $\mathrm{In}_{1-x} \mathrm{Mn}_{x} \mathrm{~S}$, the $\mathrm{Mn}$ ions go into the lattice substitutionally at the Ga or In lattice site similar to the II-VI DMS. ${ }^{2,4}$ However, the $\operatorname{In}_{1-x} \mathrm{Mn}_{x} \mathrm{~S}$ structure contains two preferred orientations of the In-In bond that are $70^{\circ}$ apart. ${ }^{4}$ There are no comparable II-VI DMS systems exhibiting this feature. Another behavior in the III-VI DMS with no comparable II-VI DMS system ${ }^{10}$ is the large thermal hysteresis between 90 and $290 \mathrm{~K}$ recently reported in $\mathrm{In}_{1-x} \mathrm{Mn}_{x} \mathrm{Se}^{6,7}$ While spin-glass transitions are known to exist in a number of systems ${ }^{10,11}$

${ }^{a)}$ Electronic mail: tpekarek@unf.edu including II-VI DMS such as $\mathrm{Zn}_{1-x} \mathrm{Mn}_{x} \mathrm{Te},{ }^{12}$ no III-VI DMS has been reported to exhibit a spin-glass transition except for $\mathrm{Ga}_{1-x} \mathrm{Mn}_{x} \mathrm{~S}$. ${ }^{13}$

The III-VI semiconductors GaSe, ${ }^{8,14-19}$ InSe, ${ }^{15,18-24}$ $\mathrm{GaTe}^{25}$ and GaS (Refs. 26-28) have received considerable interest in the last few years because of their remarkable nonlinear optical properties. Recent work on $\mathrm{GaS}$ includes growth of $\mathrm{GaS}$ films and multilayers on a GaAs substrate, ${ }^{27,29}$ thermal conductivity, ${ }^{30}$ and fabrication of Ohmic contacts to $n$-GaAs using GaS. ${ }^{28,31}$ Suhre et al. have

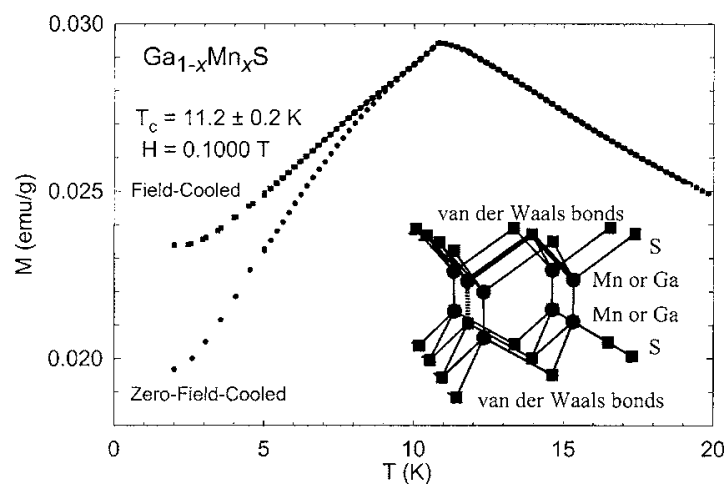

FIG. 1. Magnetization vs temperature data for $\mathrm{Ga}_{1-x} \mathrm{Mn}_{x} \mathrm{~S}$ in a $0.1 \mathrm{~T}$ field. Note the cusp near $11.2 \mathrm{~K}$ and the splitting in the field-cooled (FC) and zero-field-cooled (ZFC) traces due to a spin-glass transition. The inset shows a cross section for a four atom thick layer of $\mathrm{Ga}_{1-x} \mathrm{Mn}_{x} \mathrm{~S}$. More complex exchange channels are evident. 
shown that adding In to GaSe strengthens the material enough to polish optical faces along arbitrary crystalline directions while enhancing the optical characteristics. ${ }^{16}$

In this work we present magnetic measurements to expand our understanding of a spin-glass transition in the layered III-VI DMS Ga $a_{1-x} \mathrm{Mn}_{x} \mathrm{~S} .{ }^{13}$ Since no other III-VI DMS is currently known to exhibit a spin-glass transition, we continue to study this spin-glass system to help determine where it fits into the rich collection of other known spinglass materials. This work also complements work done on the other III-VI DMS system investigated to date $\left(\mathrm{Ga}_{1-x} \mathrm{Mn}_{x} \mathrm{~S},{ }^{1,2} \mathrm{In}_{1-x} \mathrm{Mn}_{x} \mathrm{~S},{ }^{4,5} \mathrm{In}_{1-x} \mathrm{Mn}_{x} \mathrm{Se},{ }^{6,7} \mathrm{Ga}_{1-x} \mathrm{Mn}_{x} \mathrm{Se},{ }^{8}\right.$ and $\left.\mathrm{Ga}_{1-x} \mathrm{Fe}_{x} \mathrm{Se}\right){ }^{9}$

\section{EXPERIMENTAL DETAILS}

A $0.0160 \mathrm{~g}$ single crystalline $\mathrm{Ga}_{1-x} \mathrm{Mn}_{x} \mathrm{~S}$ sample was taken from a boule grown by the vertical Bridgman method with a nominal concentration of $x=0.05$. Magnetization measurements were performed using a Quantum Design MPMS XL7 superconducting quantum interference device (SQUID) magnetometer at temperatures between 1.8 and $400 \mathrm{~K}$ in fields up to $7 \mathrm{~T}$. A Curie-Weiss fit at high temperatures gave a concentration of $x=0.091$. A concentration of $x=0.089$ was obtained from a comparison of the magnetization values over a wide range of fields up to $7 \mathrm{~T}$ and temperatures above the cusp up to $400 \mathrm{~K}$ with the values obtained from a reference sample whose actual concentration was determined by both a Curie-Weiss analysis and atomic absorption spectroscopy (AAS). We therefore take the actual concentration of our sample to be $x=0.09$. Measurements for the nonlinear scaling analysis were made for fields between 0 and $1 \mathrm{~T}$ after quenching the superconducting magnet to remove any remanent field from the magnet. Magnetization versus temperature data were taken at fixed fields beginning at a temperature below the cusp and ending above where the nonlinear term had decayed to noise level. Temperature stability was achieved to within $0.01 \mathrm{~K}$. A pure GaS crystal was measured to determine the value of the diamagnetic signal ( -3.7 $\times 10^{-7} \mathrm{emu} / \mathrm{g} \mathrm{G}$ ) due to the semiconductor host GaS, which was subtracted from the data.

\section{EXPERIMENTAL RESULTS AND DISCUSSION}

Magnetization versus temperature data for $\mathrm{Ga}_{1-x} \mathrm{Mn}_{x} \mathrm{~S}$ in $0.1000 \mathrm{~T}$ is shown in Fig. 1. The key feature is the cusp with a splitting between the field-cooled and zero-field-cooled data. Above the cusp, the magnetization is reversible and the field-cooled and zero-field-cooled data overlap. Below the transition, the spin relaxation times are sufficiently long to prevent equilibrium on the time scales of the experiment resulting in a history dependent magnetization. Additional nonlinear magnetization scaling analysis, which is a key test for spin-glass transitions, was conducted and established that the cusp observed in Fig. 1 was due to a true spin-glass transition at $T_{c}=11.2 \mathrm{~K}$ for this sample. ${ }^{13}$

The divergent negative nonlinear magnetization suppresses the high temperature side of the cusp and reduces the temperature at which the cusp appears in the magnetization even in the zero-field limit. The temperature at which the

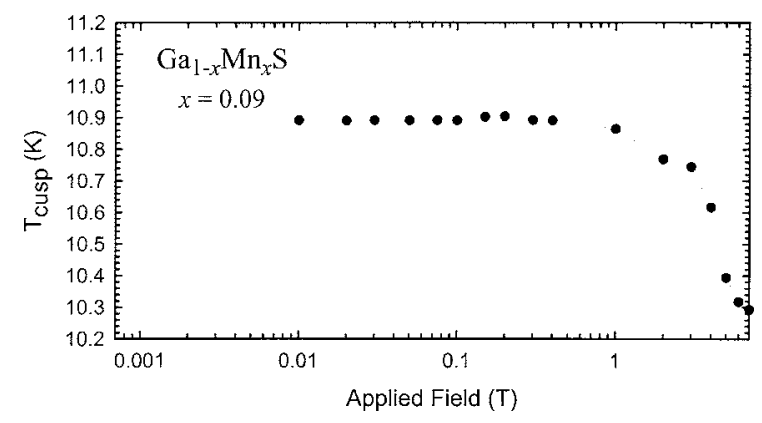

FIG. 2. Temperature of the cusp in the magnetization vs temperature data plotted vs applied field. Note the log scale on the horizontal axis. The zerofield limit persists up to $\sim 1 \mathrm{~T}$.

cusp appears in the zero-field-cooled magnetization data is plotted versus applied field in Fig. 2 for applied fields between 0.0100 and $7 \mathrm{~T}$. The temperature of the low-field spinglass transition cusp was found to occur at $10.9 \mathrm{~K}$, which is slightly lower than the true spin-glass transition temperature $T_{c}=11.2 \pm 0.2 \mathrm{~K}$ for this sample. The asymptotic, zero-field limit of the spin-glass transition cusp for $\mathrm{Ga}_{1-x} \mathrm{Mn}_{x} \mathrm{~S}$ was found to persist up to $\sim 1 \mathrm{~T}$ before being suppressed to lower temperatures with increasing field as seen in Fig. 2. This enhanced suppression of the cusp's temperature in increasing applied fields is due to the larger value of the nonlinear susceptibility $\chi_{\mathrm{nl}}=-M_{\mathrm{nl}} / H\left(M_{\mathrm{nl}}\right.$ is the nonlinear magnetization) with applied field at temperatures just above $T_{c}$.

The nonlinear magnetization was investigated in several applied fields $(130,200,300,500,750$, and $1000 \mathrm{Oe})$ and is plotted in Fig. 3 as $-M_{\mathrm{nl}} / H$ vs $T$. The linear component of the magnetization was determined between 12.7 and $15.0 \mathrm{~K}$ and is subtracted from the data. The nonlinear magnetization

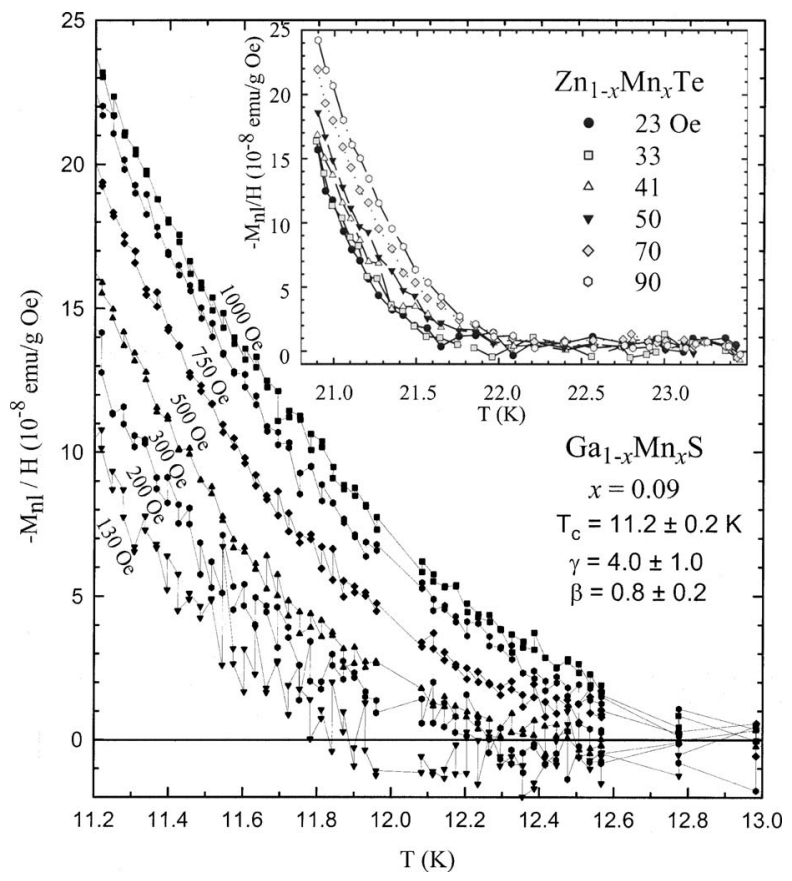

FIG. 3. Nonlinear susceptibility $-M_{\mathrm{nl}} / H$ vs temperature $T$ for $\mathrm{Ga}_{1-x} \mathrm{Mn}_{x} \mathrm{~S}$ for several fixed fields. The divergence of the nonlinear term near $T_{c}$ is expected near a spin-glass transition. The inset shows the nonlinear susceptibility $-M_{\mathrm{nl}} / H$ vs temperature for $\mathrm{Zn}_{0.49} \mathrm{Mn}_{0.51}$ Te. Note the similar scale for the $-M_{\mathrm{nl}} / H$ axis for both systems. 


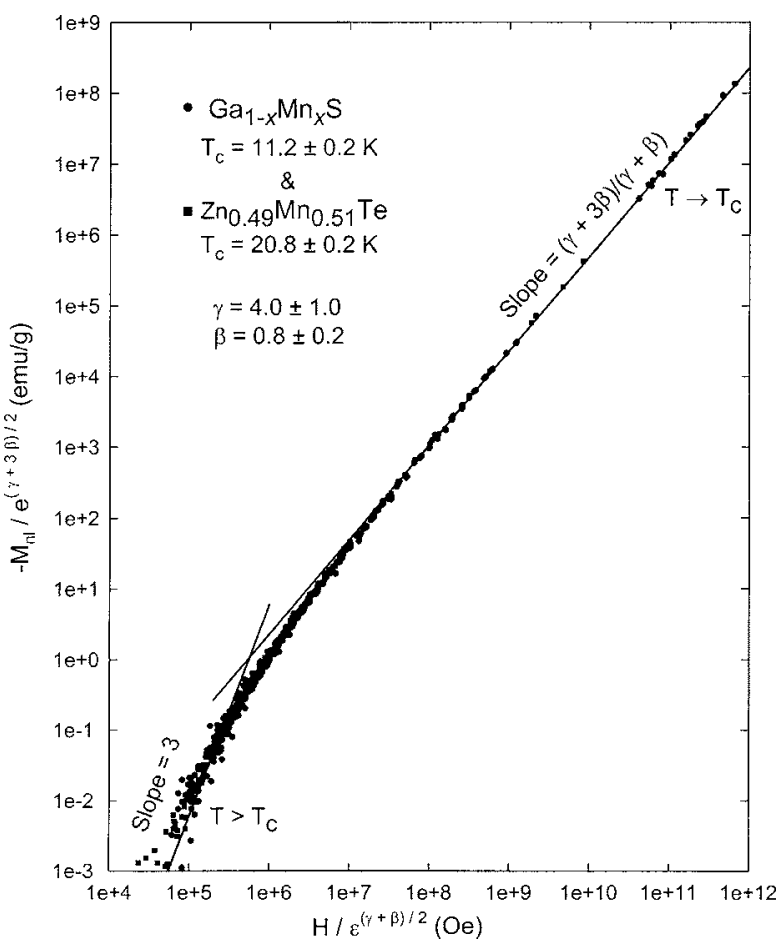

FIG. 4. Nonlinear magnetization data analyzed according to a universal scaling model for $\mathrm{Ga}_{1-x} \mathrm{Mn}_{x} \mathrm{~S}$ and $\mathrm{Zn}_{0.49} \mathrm{Mn}_{0.51} \mathrm{Te}$. Note the excellent overlap following the same universal scaling function over the entire range.

taken in $0.1000 \mathrm{~T}$ is clearly seen to diverge as the temperature approaches $T_{c}=11.2 \mathrm{~K}$. The negative divergence in the $0.1000 \mathrm{~T}$ nonlinear magnetization (Fig. 3) corresponds to the negative concavity of the cusp in Fig. 1. For comparison, the inset of Fig. 3 shows strikingly similar data (note the identical scale on the $y$ axis) taken for the II-VI DMS spin-glass system $\mathrm{Zn}_{0.49} \mathrm{Mn}_{0.51} \mathrm{Te}^{12}$

Figure 4 shows the results from a nonlinear magnetization scaling analysis for $\mathrm{Ga}_{1-x} \mathrm{Mn}_{x} \mathrm{~S}$ (Ref. 13) using the universal scaling relation $M_{\mathrm{nl}}(\varepsilon, H)=\varepsilon^{(\gamma+3 \beta) / 2} F\left(H / \varepsilon^{(\gamma+\beta) / 2}\right)$, where $\beta$ is the critical exponent for the order parameter, $\gamma$ is another critical exponent, and $\varepsilon=\left(T-T_{c}\right) / T_{c}$ is the reduced temperature. ${ }^{32,33}$ The parameter values that produced the best scaling were $\gamma=4.0 \pm 1.0, \beta=0.8 \pm 0.2$, and $T_{c}=11.2 \pm 0.2 \mathrm{~K}$. The data collapse onto the single universal scaling curve that covers many orders of magnitude along both axes. For temperatures significantly greater than $T_{c}$ (lower part of the curve), the slope approaches 3 as expected. As $T$ closely approaches $T_{c}$ from above (upper part of the curve), the slope tends to the proper asymptotic value $(\gamma+3 \beta) /(\gamma+\beta){ }^{32}$ These asymptotic limits are shown as solid lines in Fig. 4. Additional analysis is described elsewhere. ${ }^{13}$

Given the strikingly similar nonlinear magnetization data (Fig. 3) for both the $\mathrm{Ga}_{1-x} \mathrm{Mn}_{x} \mathrm{~S}$ and $\mathrm{Zn}_{0.49} \mathrm{Mn}_{0.51} \mathrm{Te}$ systems, we plotted the nonlinear magnetization for $\mathrm{Zn}_{0.49} \mathrm{Mn}_{0.51} \mathrm{Te}$ along with the $\mathrm{Ga}_{1-x} \mathrm{Mn}_{x} \mathrm{~S}$ data. Interestingly, both systems have the same critical exponent values $(\gamma=4.0 \pm 1.0$ and $\beta$ $=0.8 \pm 0.2$ ). The vertical axis for the $\mathrm{Zn}_{0.49} \mathrm{Mn}_{0.51} \mathrm{Te}$ data has been shifted downward slightly to correct for concentration differences. As can be seen, the $\mathrm{Ga}_{1-x} \mathrm{Mn}_{x} \mathrm{~S}$ system follows the same universal scaling function as $\mathrm{Zn}_{0.49} \mathrm{Mn}_{0.51} \mathrm{Te}$ and is indistinguishable from the $\mathrm{Zn}_{0.49} \mathrm{Mn}_{0.51}$ Te data on this scale.

\section{ACKNOWLEDGMENTS}

This research was supported by the Donors of the American Chemical Society Petroleum Research Fund PRF 40209-B5M, a Purdue University Academic Reinvestment Program, and by the National Science Foundation (NSF) Grants Nos. DMR-03-05653 and DMR-04-05082.

${ }^{1}$ T. M. Pekarek, M. Duffy, J. Garner, B. C. Crooker, I. Miotkowski, and A. K. Ramdas, J. Appl. Phys. 87, 6448 (2000).

${ }^{2}$ C. Fuller, A. Douglas, J. Garner, T. M. Pekarek, I. Miotkowski, and A. K. Ramdas, Phys. Rev. B 65, 195211 (2002).

${ }^{3}$ J. L. Tracy, R. Mourad, J. Garner, T. M. Pekarek, I. Miotkowski, and A. K. Ramdas, J. Appl. Phys. 99, 08D507 (2006).

${ }^{4}$ J. L. Tracy, R. Mourad, J. Garner, T. M. Pekarek, I. Miotkowski, and A. K. Ramdas, Phys. Rev. B 72, 165201 (2005).

${ }^{5}$ G. Franzese, A. Byrd, J. T. Tracy, J. Garner, T. M. Pekarek, I. Miotkowski, and A. K. Ramdas, J. Appl. Phys. 97, 10D308 (2005).

${ }^{6}$ T. M. Pekarek, L. H. Ranger, I. Miotkowski, and A. K. Ramdas, J. Appl. Phys. 99, 08D511 (2006).

${ }^{7}$ T. M. Pekarek, D. J. Arenas, I. Miotkowski, and A. K. Ramdas, J. Appl. Phys. 97, 10M106 (2005)

${ }^{8}$ T. M. Pekarek, B. C. Crooker, I. Miotkowski, and A. K. Ramdas, J. Appl. Phys. 83, 6557 (1998).

${ }^{9}$ T. M. Pekarek, C. L. Fuller, J. Garner, B. C. Crooker, I. Miotkowski, and A. K. Ramdas, J. Appl. Phys. 89, 7030 (2001).

${ }^{10}$ Semiconductors and Semimetals, edited by J. K. Furdyna and J. Kossut (Academic, Boston, 1988), Vol. 25.

${ }^{11}$ Spin Glasses: An Experimental Introduction, edited by J. A. Mydosh (Taylor \& Francis, Washington, DC, 1993).

${ }^{12}$ P. M. Shand, A. D. Christianson, T. M. Pekarek, L. S. Martinson, J. W. Schweitzer, I. Miotkowski, and B. C. Crooker, Phys. Rev. B 58, 12876 (1998).

${ }^{13}$ T. M. Pekarek, E. M. Watson, J. Garner, P. M. Shand, I. Miotkowski, and A. K. Ramdas, Phys. Rev. Lett. (submitted).

${ }^{14}$ N. C. Fernelius, Prog. Cryst. Growth Charact. Mater. 28, 275 (1994).

${ }^{15}$ A. Segura, J. Bouvier, M. V. Andrés, F. J. Manjón, and V. Muñoz, Phys. Rev. B 56, 4075 (1997).

${ }^{16}$ D. R. Suhre, N. B. Singh, V. Balakrishni, N. C. Fernelius, and F. K. Hopkins, Opt. Lett. 22, 775 (1997).

${ }^{17}$ S. Meng, B. R. Schroeder, and M. A. Olmstead, Phys. Rev. B 61, 7215 (2000).

${ }^{18}$ D. E. N. Brancus and G. Stan, Phys. Rev. B 63, 235203 (2001).

${ }^{19}$ M. O. D. Camara, A. Mauger, and I. Devos, Phys. Rev. B 65, 205308 (2002).

${ }^{20}$ I. H. Choi and P. Y. Yu, Phys. Rev. B 68, 165339 (2003)

${ }^{21}$ G. Ferlat, H. Xu, V. Timoshevskii, and X. Blase, Phys. Rev. B 66, 085210 (2002).

${ }^{22}$ S. Nüsse, P. H. Bolivar, H. Kurz, F. Levy, A. Chevy, and O. Lang, Phys. Rev. B 55, 4620 (1997).

${ }^{23}$ D. Errandonea, A. Segura, J. F. Sánchez-Royo, V. Muñoz, P. Grima, A. Chevy, and C. Ulrich, Phys. Rev. B 55, 16217 (1997).

${ }^{24}$ F. J. Manjon, D. Errandonea, A. Segura, and V. Munoz, Phys. Rev. B 63, 125330 (2001).

${ }^{25}$ J. Z. Wan, J. L. Brebner, and R. Leonelli, Phys. Rev. B 53, 15413 (1996).

${ }^{26}$ F. J. Manjón, A. Segura, and V. Muñoz, J. Appl. Phys. 81, 6651 (1997).

${ }^{27}$ A. B. M. O. Islam, T. Tambo, and C. Tatsuyama, J. Appl. Phys. 85, 4003 (1999).

${ }^{28}$ N. Okamoto, T. Takahashi, and H. Tanaka, Appl. Phys. Lett. 73, 794 (1998).

${ }^{29}$ M. Budiman, T. Okamoto, A. Yamada, and M. Konagai, Jpn. J. Appl. Phys., Part 1 37, 5497 (1997).

${ }^{30}$ M. A. Alzhdanov, M. D. Nadzhafzade, and X. Y. Seidov, Phys. Solid State 41, 20 (1999).

${ }^{31}$ N. Okamoto, T. Takahashi, T. Tanaka, and M. Takikawa, Jpn. J. Appl. Phys., Part 1 37, 3248 (1998).

${ }^{32}$ A. Mauger, J. Ferré, and P. Beauvillain, Phys. Rev. B 40, 862 (1989).

${ }^{33}$ A. P. Ramirez, G. P. Espinosa, and A. S. Cooper, Phys. Rev. Lett. 23, 2070 (1990). 